\title{
AIAA-98-1991 \\ BUCKLING AND STABLE TEARING RESPONSES OF UNSTIFFENED ALUMINUM SHELLS WITH LONG CRACKS
}

\author{
James H. Starnes, Jr. ${ }^{*}$, and Cheryl A. Rose ${ }^{\dagger}$ \\ NASA Langley Research Center \\ Hampton, Virginia 23681-001
}

\begin{abstract}
The results of an analytical and experimental study of the nonlinear response of thin, unstiffened, aluminum cylindrical shells with a long longitudinal crack are presented. The shells are analyzed with a nonlinear shell analysis code that accurately accounts for global and local structural response phenomena. Results are presented for internal pressure and for axial compression loads. The effect of initial crack length on the initiation of stable tearing and unstable crack growth in typical shells subjected to internal pressure loads is predicted using geometrically nonlinear elastic-plastic finite element analyses. The results of these analyses and of the experiments indicate that the pressure required to initiate stable tearing and unstable tearing in a shell subjected to internal pressure loads decreases as the crack length increases. The effects of crack length on the prebuckling, buckling and postbuckling responses of typical shells subjected to axial compression are also described. For this loading condition, the crack length is held constant. The results of the analyses illustrate the influence of crack length on shell buckling instabilities. The experimental and analytical results correlate well.
\end{abstract}

\section{Introduction}

Transport fuselage shell structures are designed to support combinations of internal pressure and mechanical loads which can cause the structure to have a geometrically nonlinear structural response. These shell structures are required to have adequate structural integrity so that they do not fail if cracks develop during the service life of the aircraft. The structural response of a transport fuselage shell structure with a crack is influenced by the local stress and displacement gradients near the crack and by the internal load distribution in the shell. Local fuselage out-of-plane skin displacements near a crack can be large compared to the fuselage skin thickness, and these displacements can couple with the internal stress resultants in the shell to amplify the magnitudes

\footnotetext{
Head, Structural Mechanics Branch. Fellow, AIAA.

Aerospace Engineer, Structural Mechanics Branch. Member, AIAA.

Copyright 01998 by the American Instinte of Aeronautics and Astronautics, Inc. No copyright is asserted in the United States under Title 17, U. S. Code. The U. $\mathrm{S}$. Government has a royalty-free license to exercise all rights under the copyright S. Government has a royalty-free license to exercise all rights under the copyright
claimed herein for Governmental Purposes. All other rights are reserved by the copyright owner.
}

of the local stresses and displacements near the crack. In addition, the stiffness and internal load distributions in a shell with a crack will change as the crack grows and when the skin buckles. These changes will affect the local stress and displacement gradients near the crack. This nonlinear response must be understood and accurately predicted in order to determine the structural integrity and residual strength of a fuselage structure with damage. Recent studies (e.g., Refs. 1-5) have shown that the structural response and structural integrity of a shell with a crack can be studied analytically with a nonlinear structural analysis procedure that can model crack growth in the shell.

Typical nonlinear analysis results presented in Refs. 3 and 4 indicate that different combinations of applied loads can cause different responses of a stiffened shell with a long crack. The magnitudes of the mechanical loads used in the studies described in Refs. 3 and 4 are representative of loads that do not buckle the skin of the fuselage. Fuselage shells are usually designed to allow the fuselage skin to buckle above a specified design load that is less than the design limit load for the shell. During the design of the fuselage, it is assumed that the design limit load can occur anytime during the service life of the aircraft. As a result, a long crack could exist in the fuselage shell after a considerable amount of flight service, and loading conditions could occur that cause the shell with the long crack to buckle. The results of a preliminary analytical study ${ }^{5}$ of the effects of long cracks on the nonlinear response of unstiffened aluminum shells indicates that the behavior of a shell can be influenced significantly by the initial length of the crack .

The present paper describes the results of an analytical and experimental study of the effects of internal pressure and axial compression loads on the responses of thin, unstiffened, laboratory scale, aluminum cylindrical shells with a long longitudinal crack. Two wall thicknesses are considered in the analytical study to determine the effect of wall thickness on the shell response. The predicted effect of the initial crack length on the initiation of stable tearing and on unstable crack growth is discussed for shells subjected to internal pressure loads. In addition, predicted prebuckling, buckling, and initial postbuckling results are presented and compared for cy-

1

American Institute of Aeronautics and Astronautics 
lindrical shells subjected to axial compression loads. The results presented illustrate the influence of the loading condition and crack length on shell crack-growth instabilities, and on shell buckling instabilities.

\section{Shell Model and Analysis Procedure}

\section{Shell Model}

The geometry of the shells analyzed in this study is defined in figure la. The shells have a 9.0-inch radius, $R$, a 0.040 - or 0.020 -inch-thick wall, $t$, and a 36.0-inch length, $L$. A longitudinal crack is located at $\theta=0^{\circ}$ and at shell midlength. The initial crack length, $a$, ranges from 1.0 to 4.0 inches. The shells are typical laboratory-scale cylindrical shells and are made of 2024-T3 aluminum alloy. The Young's modulus, E, for the aluminum alloy is equal to $10 \mathrm{msi}$ and Poisson's ratio is equal to 0.3 . The yield stress for the material is $50 \mathrm{ksi}$ and the ultimate stress is $72 \mathrm{ksi}$.

\section{Nonlinear Analysis Procedure}

The shell responses were studied numerically using the STAGS (STructural Analysis of General Shells) nonlinear shell analysis code. ${ }^{6}$ STAGS is a finite element code designed for the static and dynamic analysis of general shells and includes the effects of geometric and material nonlinearities in the analysis. The code uses both the modified and full Newton methods for its nonlinear solution algorithms, and accounts for large rotations in a shell by using a co-rotational algorithm at the element level. STAGS has static and transient analysis capabilities that can be used to predict local instabilities and modal interactions that occur due to destabilizing mechanical loads, such as an applied compression or shear load. The Riks pseudo arc-length path following method $^{7,8}$ is used to continue a solution past the limit points of a nonlinear response. With this strategy, the incrementally applied loading parameter is replaced by an arc-length along the solution path which is then used as an independent loading parameter. The arc-length increments are automatically adjusted by the program as a function of the solution behavior.

STAGS can also perform crack-propagation analyses, and can represent the effects of crack growth on nonlinear shell response. A nodal release method and a loadrelaxation technique are used to extend a crack while the shell is in a nonlinear equilibrium state. ${ }^{2}$ The condition for crack extension is based upon a fracture criterion. When a crack is to be extended, the forces necessary to hold the current crack tip nodes together are calculated. The crack is extended by releasing the nodal compatibility condition at the crack tip, applying the equivalent crack-tip forces, and then releasing these forces to establish a new equilibrium state, which corresponds to the longer crack. The changes in the stiffness matrix and the internal load distribution that occur during crack growth are accounted for in the analysis, and the nonlinear cou- pling between internal forces and in-plane and out-ofplane displacement gradients that occurs in a shell are properly represented. Output from STAGS, associated with a crack, includes the strain-energy-release rate in an elastic analysis, and the crack-tip opening angle (CTOA) in an elastic-plastic analysis. ${ }^{2.9}$ These quantities can then be used as part of a fracture criterion in an elastic analysis or an elastic-plastic analysis to predict the residual strength and stable crack growth behavior of a damaged shell.

The axial compression loads and internal pressure loads were modeled in STAGS using two separate load sets. For the case of axial compression only, the compression load was applied to the ends of the shell by specifying a uniform end displacement. For the internal pressure load cases, internal pressure was simulated by applying a uniform lateral pressure to the shell wall and applying an axial tensile force to the ends of the shell with multi-point constraints to enforce a uniform end displacement. The loading conditions were applied to the finite element models shown in figure 1. Both the standard 410 element and the 510 transition element from the STAGS element library were used in the models. Radial displacement constraints were imposed on the ends of the shells to insure that the ends of the shells remain circular during the load application.

For the internal pressure load case, advantage was taken of the symmetry of the problem, and only a quarter of the shell was modeled, as shown in figure 1b. Symmetry conditions were applied along the edges $\theta=0^{\circ}$ and $\theta=-180^{\circ}$ and along the edge $x=18$ inches. Cracks with initial half-lengths of 0.5 to 2.0 inches were defined in the model along $\theta=0^{\circ}$ and extended from the symmetry boundary at the edge, $x=18$ inches. Growth of the cracks was studied for a quasi-static increase in the magnitude of the internal pressure loading. An elastic-plastic analysis, with a critical crack-tip opening angle (CTOA) fracture criterion, ${ }^{9,10}$ was used to simulate the stable crack growth in the shell. In addition, straight cracks were assumed, so that crack extension was self-similar. The White-Besseling theory, with a piece-wise linear stress-strain curve, ${ }^{11}$ was used to represent the material nonlinearity. A high level of mesh refinement was required around the crack tip to predict accurately the yielding at the crack tip, and crack extension. Crack extension was assumed to occur when the crack-tip opening angle was equal to $5.36^{\circ}$.

The entire cylindrical shell was modeled for the axial compression load case. The finite element model for this load cases is shown in figure 1c. Straight cracks with lengths of 1.0 to 4.0 inches were defined in the model along $\theta=0^{\circ}$ and at the shell midlength. Plasticity effects were included for some of the analyses for this load case. The primary objective of the study for the axial compression case was to continue the analysis beyond the critical buckling state, and to focus on the postbuck- 
ling response of the shell with a crack. Thus, the interaction between local buckling and crack extension was not specifically addressed; that is, the crack lengths were held constant throughout the analyses.

The prebuckling, buckling and postbuckling responses of the shells for the axial compression loading condition were determined using the following analysis procedure. The prebuckling responses were determined using the geometrically nonlinear quasi-static analysis capability in STAGS. The Riks pseudo arc-length path following method, described above, was used to compute the initial shell response just after buckling. The initial, unstable, postbuckling response of the shell was predicted using the nonlinear transient analysis option of the code. The transient analysis was initiated from an unstable equilibrium state just beyond the buckling point by incrementing the end displacement. The transient analysis was continued until the transient response damped out or decayed. Once the transient analysis converged to a steady-state solution, the load relaxation option of the code was used to establish a stable equilibrium state. The subsequent stable postbuckling response of the shell was computed using the standard nonlinear, static analysis option.

\section{Fracture Criterion}

As mentioned in the previous section, crack extension for the pressure loading case was simulated using the CTOA fracture criterion. The CTOA fracture criterion uses the crack opening angle, measured at a fixed distance from the crack tip, as the fracture parameter. The criterion assumes that stable crack growth will occur when the angle reaches a critical value. The critical angle for a particular material and thickness can be obtained by numerically simulating the fracture behavior of a laboratory specimen, using an elastic-plastic finite element analysis, and determining the angle that best describes the experimentally observed fracture behavior. The determined angle can then be used to predict the fracture behavior of different structural configurations. In the numerical simulation, a characteristic distance behind the crack tip is defined for evaluating the angle. The crack opening displacements behind the crack are monitored and the crack is advanced by one element length when the opening angle evaluated at the characteristic distance reaches the critical value. In the present study, the characteristic distance was equal to 0.04 inches (minimum element size along the line of crack extension). Newman ${ }^{9}$ has shown this value to be adequate for analyzing stable tearing in a wide variety of materials. The critical angle of $5.36^{\circ}$ used in this study, is a preliminary value that was determined by matching STAGS predictions for the fracture behavior of a shell with the geometry described above and with a 4.0-inch-long longitudinal crack, with the experimentally observed behavior of an aluminum shell of the same geometry and subjected to internal pressure.

\section{Experiments}

\section{Test Specimens}

The specimens tested in this investigation were fabricated from 0.040 -inch-thick 2024-T3 bare aluminum alloy sheet. All specimens were 39 inches long and 18 inches in diameter. Each specimen was fabricated with a 1.5-inch-wide double lap splice with 0.040 -inch-thick splice plates and a single row of 0.1875 -inch-diameter aluminum rivets on each side of the splice. The rivets were spaced 1.5 inches apart along the length of the specimens and were located five rivet diameters from the edges of the splice plates. Longitudinal cracks were machined in the specimens at the mid length of the specimens, and the cracks were diametrically opposed to the splice in the specimen. The cracks were 0.010 inches wide and either $2.0,3.0$ or 4.0 inches long. Specimens subjected to internal pressure loads had the crack tips sharpened with a sharp knife.

\section{Apparatus and Tests}

Both ends of each specimen were potted in an aluminum-filled epoxy resin to assure proper load introduction during the tests. The potting material extended 1.5 inches along the length of the specimens at each end, and the test section of the specimens was 36 inches long. Specimens subjected to compression loads were machined flat and parallel, and tested in a 300-kip hydraulic testing machine. Specimens subjected to internal pressure loads were attached to end fixtures, and slowly pressurized to failure with nitrogen gas. One end of the pressurized specimens was unconstrained in the axial direction to allow free expansion of the specimen. All specimens were instrumented with electrical resistance strain gages and direct current differential transducers were used to measure displacements. Crack wire gages were applied at a crack tip of the specimens subjected to internal pressure loads to record the stable tearing of the crack. A moiré interferometry procedure was used with the specimens subjected to axial compression loads to observe the deformation patterns before and after buckling had occurred. Three non-collinear direct current differential transducers were used to measure the displacements of the loading platen for the compression loaded specimens. All data were recorded with a data acquisition system, and the response of all specimens was recorded on video tape.

\section{Results and Discussion}

The nonlinear analysis and test results for thin unstiffened aluminum cylindrical shells with a longitudinal crack are presented in this section. Results have been generated for two loading conditions: internal pressure only, and axial compression only. Results for these loading conditions are presented for shells with a longitudinal crack at shell midlength and with initial crack lengths of 
1.0, 2.0, 3.0 and 4.0 inches. The maximum value of the applied internal pressure considered is $143 \mathrm{psi}$. This pressure is the pressure required to cause a shell with a 1.0-inch-long initial crack and a 0.040 -inch wall thickness to fail due to an internal pressure load. The axial compression loads are increased from zero to the maximum axial load that the shell can support. Typical results are presented to illustrate the effects of crack length on the tearing response of a shell subjected to internal pressure loads, and on the prebuckling, buckling and postbuckling responses of a shell subjected to axial compression loads. Shells with both 0.020 - and 0.040 - inch wall thicknesses are considered for the analysis results, but only shells with 0.040 -inch wall thickness are considered for the experimental results.

\section{Internal Pressure Loads}

The effect of increasing the internal pressure in a shell on the total crack growth or crack extension is shown in figures $2 \mathrm{a}$ and $2 \mathrm{~b}$ for shells with initial crack lengths of 1.0, 2.0,3.0, and 4.0 inches, and with wall thicknesses of 0.040 and 0.020 -inches, respectively. These results indicate that the internal pressure in the shell can be increased, and the cracked shell will remain in equilibrium, up to a pressure at which yielding occurs at the crack tips and the opening angle at the crack tips reaches a critical value. At this pressure, the crack will start to grow. The initial growth of the crack is stable and the crack will not extend unless the pressure is increased. Eventually, unstable crack growth occurs. Unstable growth occurs when the slope of the curves in figures $2 \mathrm{a}$ and $2 b$ becomes zero, which means that a small increase in pressure causes a very large increment in crack extension.

The effect of increasing the initial crack length on the internal pressure required to initiate stable tearing and on the pressure at which stable growth changes to unstable crack growth is summarized in figure $2 \mathrm{c}$ for the two shell wall thicknesses. The lower curve for each wall thickness represents the pressure required to initiate stable tearing as a function of normalized initial crack length, and the upper curve for each wall thickness represents the pressure that causes the crack growth to become unstable. These results indicate that the internal pressure required to initiate stable tearing and to cause unstable crack growth decreases as the initial crack length increases. The results shown in figure 2 also indicate that the difference between the internal pressure required to initiate stable tearing and the internal pressure that causes unstable tearing decreases as the initial crack length increases. For an initial crack length of 1.0 inch, stable tearing initiates in the 0.040 -thick shell when the internal pressure is approximately 113 psi, and unstable crack growth occurs when the internal pressure is approximately $143 \mathrm{psi}$. The difference between the internal pressure required to initiate stable tearing and the inter- nal pressure that causes unstable crack growth is approximately 30 psi for the 1.0 -inch initial crack length in the 0.040 -inch-thick shell. The results for an initial crack length of 2.0 inches in the 0.040 -inch-thick shell indicate that stable tearing initiates when the internal pressure is approximately 51 psi and unstable crack growth occurs when the internal pressure is approximately 79 psi. The difference between the internal pressure required to initiate stable tearing and the internal pressure that causes unstable crack growth for this initial crack length is 28 psi. The difference between the internal pressure required to initiate stable tearing and the internal pressure that causes unstable crack growth for the 3.0- and 4.0inch initial crack lengths in the 0.040 -inch-thick shell is approximately 24 psi and 21 psi, respectively. The results for the 0.020-inch-thick shell are similar, but with lower values of internal pressure.

The results from the nonlinear analyses indicate that the internal pressure load induces large outward radial displacements in the neighborhood of the crack. The response associated with these radial displacements is often referred to as "crack bulging" in the literature and is the cause of the larger crack opening displacements and crack-tip stress-intensity factors in a shell, compared to those for a flat sheet under otherwise identical conditions. $^{12}$ Furthermore, the extent of crack bulging is a function of the nondimensional crack curvature parameter, $a / \sqrt{r t}$. This observation is illustrated for the 0.040 inch-thick shell in figure 3 , which shows the radial displacement response along the shell length at the circumferential location $\theta=0^{\circ}$, just before the initiation of stable crack growth, for each of the initial crack lengths. The radial displacement at the center of the crack normalized by the shell thickness, $t$, is $w_{o} / t=1.00,1.65$, 2.38 and 3.23 for the 1.0-, 2.0- 3.0- and 4.0-inch-long cracks, respectively. These displacements are greater than or equal to the shell wall thickness for all of the crack lengths considered, and represent large displacements in the context of nonlinear thin shell theory. The increase in crack bulging for the longer crack lengths is consistent with the observation that the pressure required to initiate stable tearing decreases with the increase in crack length.

Experimental and analytical results for the 0.040 inch-thick shell are compared in figure 4 for shells with initial crack lengths of $2.0,3.0$, and 4.0 inches. As previously mentioned, the experimental data obtained for the shell with a 4.0-inch-long initial crack were used to determine the critical CTOA angle to be used in predicting the response of the shells with 2.0 - and 3.0-inch-long cracks. The analytical and experimental results correlate very well for the shells with the 2.0 - and 3.0 -inch-long initial crack lengths.

\section{Axial Compression Loads}

The predicted load-shortening responses for a 
0.040 -inch-thick shell and a 0.020 -inch-thick shell with initial crack lengths of 1.0, 2.0,3.0 and 4.0 inches and subjected to axial compression are shown in figures 5 a and $5 b$, respectively. An initial outward geometric imperfection in the form of the lowest eigenmode was used in the nonlinear analyses to trigger local deformations in the vicinity of the crack. The applied compression and end-shortening values are normalized by the corresponding classical buckling values for a shell without a crack. For the 0.040-inch-thick shell and the 0.020-inch-thick shell with a 1.0-inch-long crack, the crack introduces an effective imperfection that causes general instability to occur at the loads indicated by the $\mathbf{X}$ in figures $5 \mathrm{a}$ and $5 \mathrm{~b}$. These shells cannot support additional compression load after buckling. For a shell with a longer crack, local buckling near the crack precedes shell collapse. The filled symbols in figures $5 \mathrm{a}$ and $5 \mathrm{~b}$ identify the loads that correspond to initial local buckling near the crack for the 0.040 -inch-thick shell and the 0.020 -inch-thick shell, respectively, with the 2.0-, 3.0-, and 4.0-inch-long initial cracks. Prior to buckling the radial displacement $w_{o}$ at the center of the crack edges is nearly equal to zero. Once the critical load is reached, $w_{0}$ grows rapidly with increase in load. Initial local buckling is followed by a stable postbuckling response and the load can be further increased after local buckling has occurred near the crack edges.

As the load is increased after initial local buckling has occurred, the 0.040 -inch-thick shells with the 3.0 and 4.0-inch-long cracks, and the 0.020-inch-thick shells with the 2.0-, 3.0-, and 4.0-inch-long cracks experience a change in the local buckling mode. The initial postbuckling response of the shells after the mode change is unstable, and as a result, the axial load decreases after buckling occurs. The unstable transition region in the response predictions is indicated by the broken lines in figures $5 \mathrm{a}$ and $5 \mathrm{~b}$. The unstable transition from the stable initial buckled configuration to the stable postbuckling configuration was determined by using the transient analysis capability in STAGS. The transient analysis was continued until the kinetic energy in the system was small. A time history of the kinetic energy during the transient analysis of the 0.040 -inch-thick shell with a 3.0-inch-long crack is shown in figure 6. The deformed shapes labeled A, B, C and D in figure 6 correspond to the points $A, B, C$ and $D$ on the load-end shortening and kinetic energy history curves, and show the development of the shell's postbuckled response. Point A corresponds to the initial buckling deformation, points $\mathrm{B}$, and $\mathrm{C}$, correspond to solutions obtained during the transient analysis, and point $D$ represents the stable postbuckled equilibrium state. Once a stable equilibrium state was determined from the transient analysis, the nonlinear static analysis was resumed to compute the stable postbuckling equilibrium response results shown in figure 5a. The analysis was continued for the shells with the
3.0- and 4.0-inch-long cracks for the 0.040-inch-thick shells, and for the shells with the 2.0-, 3.0-, and 4.0-inchlong cracks for the 0.020 -inch-thick shells until computational difficulties were encountered. The point of computational difficulty is assumed to correspond to shell collapse. Collapse of the 0.040 -inch-thick and of the 0.020-inch-thick shells with 2.0-, 3.0- and 4.0-inchlong cracks is identified by the unfilled symbols in figures $5 \mathrm{a}$ and $5 \mathrm{~b}$, respectively.

The initial local buckling load predictions and qualitative approximations for the shell collapse load for the 0.020 - and 0.040 -inch-thick shells are summarized in figure $5 \mathrm{c}$. The values of the normalized initial buckling loads for the 0.040 -inch-thick shells are $P / P_{c r}=0.88$, $0.54,0.39,0.32$, and the qualitative approximate values of the normalized collapse loads are $P / P_{c r}=0.88$, $0.59,0.49,0.47$ for the 1.0-, 2.0-, 3.0- and 4.0-inch-long cracks, respectively. The values of the normalized initial buckling loads for the 0.020-inch-thick shells are $P / P_{c r}=0.73,0.41,0.30,0.24$, and the qualitative approximate values of the normalized collapse loads are $P / P_{c r}=0.73,0.47,0.43,0.41$ for the $1.0-, 2.0-, 3.0$ and 4.0-inch-long cracks, respectively. These results indicate that the magnitudes of the initial buckling loads and collapse loads for the shells decrease as the initial crack length increases. In addition, the difference between the load at initial buckling and the load at collapse is smaller for the shorter crack lengths. The initial local buckling load results for the 0.020- and 0.040-inch-thick shells are represented very well by a characteristic curve that is based on the curvature parameter $a / \sqrt{r t}$.

As the initial crack length increases, the radial displacements, $w$, near the crack increase. Radial displacement profiles along the shell length are compared in figure 7 for loads just before unstable buckling at the circumferential location $\theta=0^{\circ}$. The displacements are normalized by the shell wall thickness. The value of the radial displacement at the crack center, $w_{o}$, normalized by the shell wall thickness, is $w_{o} / t=0.2,1.2,2.1$ and 3.0 for the 1.0-, 2.0-3.0- and 4.0-inch-long cracks, respectively. These prebuckling displacements are greater than the shell thickness for crack lengths greater than or equal to 2.0 inches, and represent large displacements in the context of nonlinear thin shell theory.

Typical results of the elastic prebuckling, buckling, and postbuckling calculations are presented in figure 8 for the 0.040 -inch-thick shell with a 3.0-inch-long longitudinal crack. Resultant stress distribution contour plots obtained from the nonlinear analysis are shown on the corresponding deformed shapes in the figures. The displacement magnification factor for all images is five. In addition, on all contour plots, the darker regions of the figures represent higher values of the stress resultants, and the lighter regions represent lower values of the stress resultants. As described previously, the initial local buckling of the 0.040 -inch- thick shells with the long- 
er 3.0- and 4.0-inch-long cracks is followed by a change in the local buckling mode and then a stable postbuckling response before the shell buckles into its general instability mode. A typical example of the initial local buckling displacement pattern for a 0.040 -inch-thick shell with a 3.0- inch-long crack is shown in figure 8a. The initial buckled deformation pattern, shown in figure $8 \mathrm{a}$, is typical of the shape of the initial buckled deformation pattern for the shells with the 2.0-, 3.0- and 4.0-inch-long cracks. The primary difference in the initial buckled deformation patterns for a shell with different crack lengths, with the exception of the 1.0-inch-long crack, is the magnitude of the deformations (see figure 7). The hoop and axial stress resultant distributions indicate that the crack has a significant and noticeable effect on the prebuckling stress distribution. The stable postbuckling deformation shape, after the local mode change, is shown in figure $8 \mathrm{~b}$. The deformation pattern in figure $8 \mathrm{~b}$ indicates that the shell deforms into a local pattern with high circumferential curvature that apparently stiffens the skin near the crack enough longitudinally to stabilize the shell and to increase the amount of axial compression load that can be supported by the shell after the local mode change occurs.

The effects of material nonlinearities on the predicted load-shortening response and deformation patterns or a 0.040 -inch-thick shell with a 3.0 -inch-long crack are shown in figure 9. Initial plastic yielding occurs for an applied load corresponding to $P / P_{c r}=$ 0.43 , which is approximately 90 percent of the buckling load at the local mode change. The load-shortening predictions based upon an elastic analysis and an elasticplastic analysis, and the deformation pattern prediction just prior to the local mode change, indicate that the shell response prior to the local mode change is adequately predicted by an elastic analysis. The initial postbuckled response predictions of the two analyses are, however, significantly different. The initial postbuckled deformation pattern prediction from the elastic-plastic analyses is shown in figure $9 \mathrm{c}$ and the initial postbuckled deformation pattern prediction from the elastic analysis is shown in figure 9d. Computational difficulties were encountered in the elastic-plastic analysis at this point, preventing prediction of the response further into the postbuckling load range. The postbuckled deformation pattern predicted by the elastic-plastic analysis is very similar to the deformation pattern labeled $\mathbf{C}$ in the elastic, transient analysis shown in figure 6 . These results suggest that for the cylinder studied, yielding of the aluminum will prevent the postbuckled deformation pattern predicted by the elastic analysis from developing. This suggestion is consistent with the experimentally observed behavior. The initial postbuckled deformation pattern for the 0.040 -inch-thick cylinder with a 3.0-inchlong crack is very similar to the postbuckled deformation pattern predicted by the elastic-plastic analysis.
The predicted prebuckling and initial buckling analysis results and the measured results for the 0.040 inch-thick shells with crack lengths of 2.0, 3.0, and 4.0 inches are shown in figures $10 \mathrm{a}$ and $10 \mathrm{~b}$, respectively. Buckling of each of the shells is identified by the filled symbols in the figures, and the values of $u / u_{c r}$ and $P / P_{c r}$ at buckling are provided in parentheses. The analysis predictions were obtained using a material nonlinear analysis. The measured loading-platen displacements were used to determine the amount of end rotations of the shell, and these data were used as input loading parameters for the nonlinear analysis. The results shown in figures $10 \mathrm{a}$ and $10 \mathrm{~b}$ indicate that the analytical results slightly underestimate the experimental results for the initial buckling loads. The analytical underestimation of the initial buckling results could be a result of uncertainties in the loading platen end rotations, and other differences in the analytical model and the astested specimens. An indication of the sensitivity of the predicted initial buckling load to variations in the input end rotations is provided in figure 10c. Figure 10c compares the measured load-shortening response for a shell with a 3.0-inch-long crack with analysis results that were obtained using two different values for the end rotation about one of the loading platen axes. These results indicate that the end rotations have some effect on the magnitude of the predicted buckling loads.

\section{Concluding Remarks}

The results of an analytical study of the effects of a longitudinal crack on the nonlinear response of thin, unstiffened, aluminum cylindrical shells subjected to internal pressure and axial compression loads are presented. The results indicate that the nonlinear interaction between the in-plane stress resultants and the out-of-plane displacements near a crack in a thin shell can significantly affect the structural response of the shell. Large local stress and displacement gradients exist near a crack in a shell for all loading conditions considered in the study. The results indicate that the nonlinear response of a shell depends on the loading condition applied to the shell and the initial crack length. The magnitude of the internal pressure required to initiate stable tearing in a shell subjected to internal pressure decreases as the initial crack length increases. The magnitude of the internal pressure required to cause unstable crack growth in a shell also decreases as the initial crack length increases. The initial buckling load of a shell subjected to axial compression decreases as the initial crack length increases. Initial buckling causes general instability or collapse of a shell for shorter initial crack lengths. Initial buckling is a stable local response mode for longer initial crack lengths. This stable local buckling response is followed by a stable postbuckling response, which is followed by general or overall instability of the shell. 


\section{References}

'Riks, E., "Bulging Cracks in Pressurized Fuselages: A Numerical Study," NLR MP 87058 U, NLR National Aerospace Laboratory, The Netherlands, September 1987.

${ }^{2}$ Rankin, C. C., Brogan, F. A., and Riks, E., "Some Computational Tools for the Analysis of Through Cracks in Stiffened Fuselage Shells," Computational Mechanics, Springer International, Vol. 13, No. 3, December 1993, pp. 143-156.

${ }^{3}$ Starnes, J. H., Jr., Britt, V. O., and Rankin, C. C., "Nonlinear Response of Damaged Stiffened Shells Subjected to Combined Internal Pressure and Mechanical Loads," AIAA Paper 95-1462, April 1995.

${ }^{4}$ Starnes, J. H., Jr., Britt, V. O., Rose, C. A., and Rankin, C., C., "Nonlinear Response and Residual Strength of Damaged Stiffened Shells Subjected to Combined Loads," AIAA Paper No. 96-1555, April 1995.

${ }^{5}$ Starnes, J., H., and Rose, Cheryl A. "Nonlinear Response of Thin Cylindrical Shells with Longitudinal Cracks and Subjected to Internal Pressure and Axial Compression Loads," AIAA Paper No. 97-1144, April 1997.

${ }^{6}$ Brogan, F. A., Rankin, C. C., and Cabiness, H. D., "STAGS User Manual," Lockheed Palo Alto Research Laboratory, Report LMSC P032594, 1994.

${ }^{7}$ Riks, E., "Some Computational Aspects of the Stability Analysis of Nonlinear Structures," Computational Methods in Applied Mechanics and Engineering, Vol. 47, 1984, pp. 219-259.

${ }^{8}$ Riks, E., "Progress in Collapse Analysis," Journal of Pressure Vessel Technology, Vol. 109, 1987, pp. 2741.

${ }^{9}$ Newman, J. C., Jr., "An Elastic-Plastic Finite Element Analysis of Crack Initiation, Stable Crack Growth and Instability," ASTM STP 833, 1984, pp. 93-117.

${ }^{10}$ Newman, J. C., Jr., Dawicke, D. S., Sutton, M. A. and Bigelow, C. A., "A Fracture Criterion for Widespread Cracking in Thin-Sheet Aluminum Alloys," Proceedings of the ICAF 17th Symposium, 1993.

${ }^{11}$ Dawicke, D. S., Sutton, M. A., Newman, J. C., Jr., and Bigelow, C. A., "Measurement and Analysis of Critical CTOA for an Aluminum Alloy Sheet," NASA TM109024, September, 1993.

${ }^{12}$ Riks, E., Brogan, F. A., and Rankin, C. C., "Bulging of Cracks in Pressurized Fuselages: A Procedure for Computation," in Analytical and Computational Models of Shells, Noor, A. K., Belytschko, T., and Simo, J. C., Editors, The American Society of Mechanical Engineers, ASME-CED Vol. 3, 1989.

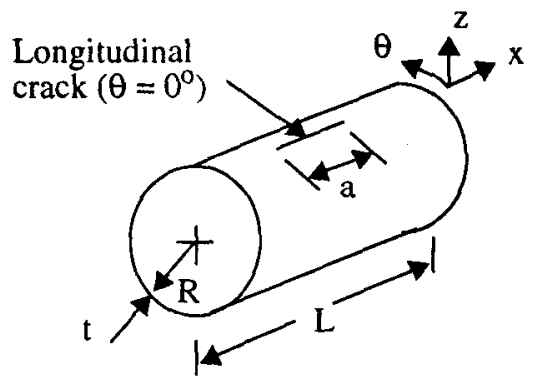

(a) Shell geometry

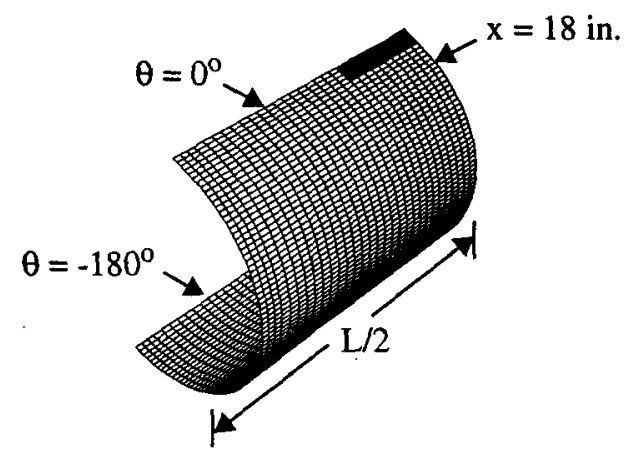

(b) Finite element model for pressure loads

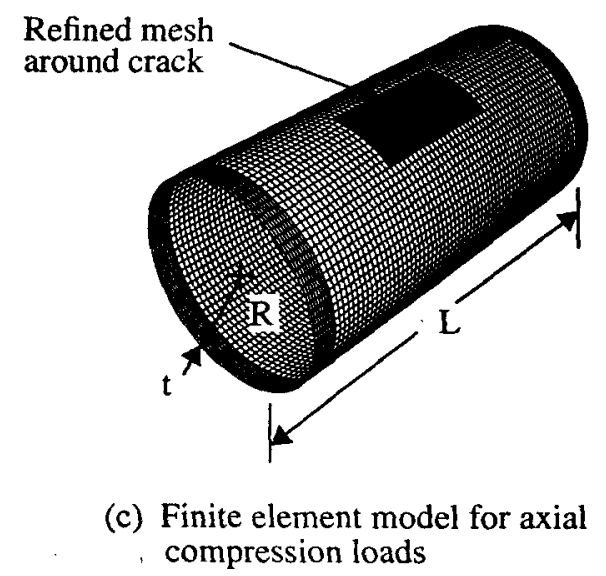

Figure 1. Shell geometry and finite element models. 


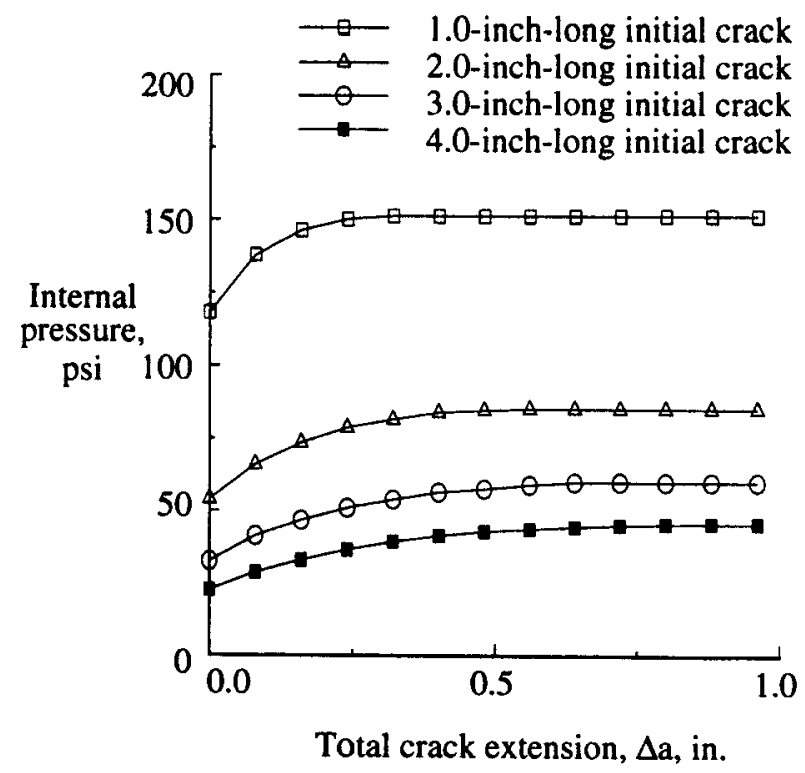

(a) Crack extension for 0.040-inch-thick shells

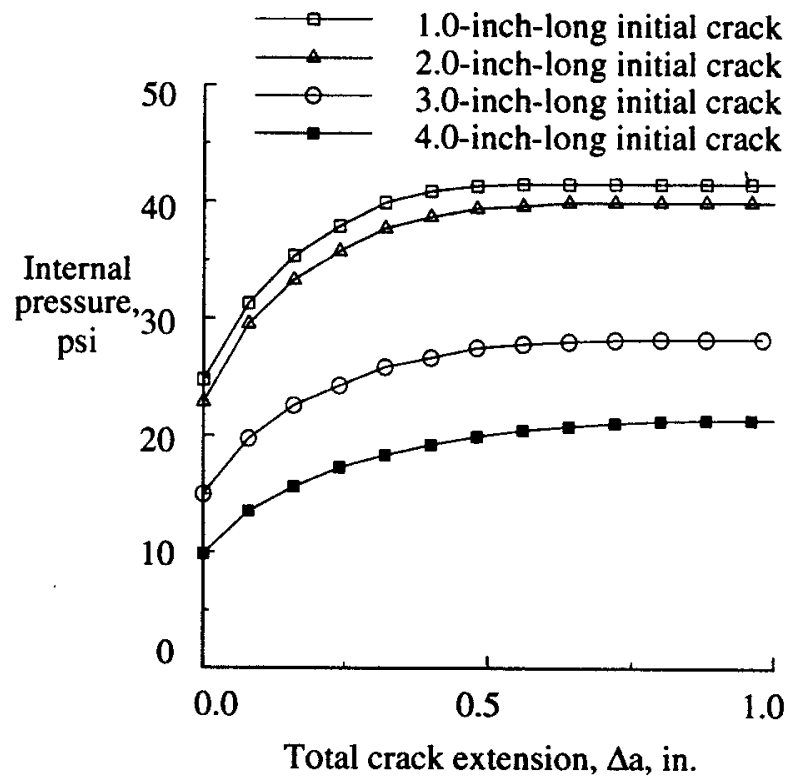

(b) Crack extension for 0.020-inch-thick shells

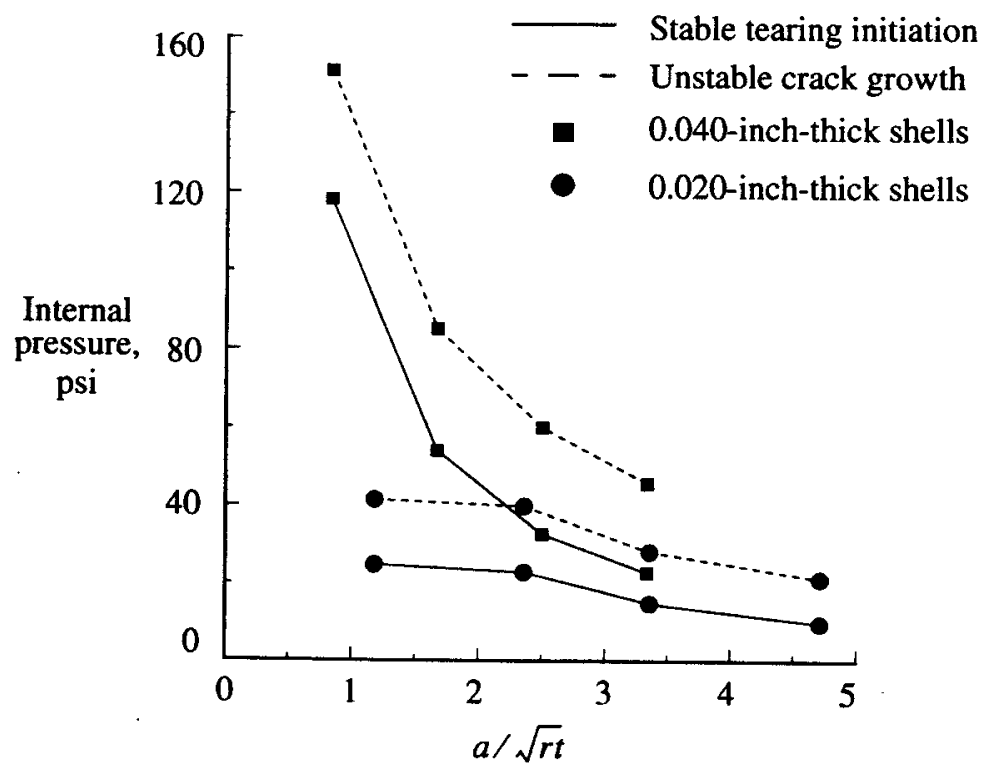

(c) Initial stable tearing and unstable crack growth

Figure 2. Effect of increasing internal pressure on initial stable tearing and unstable crack growth for different initial crack lengths. 


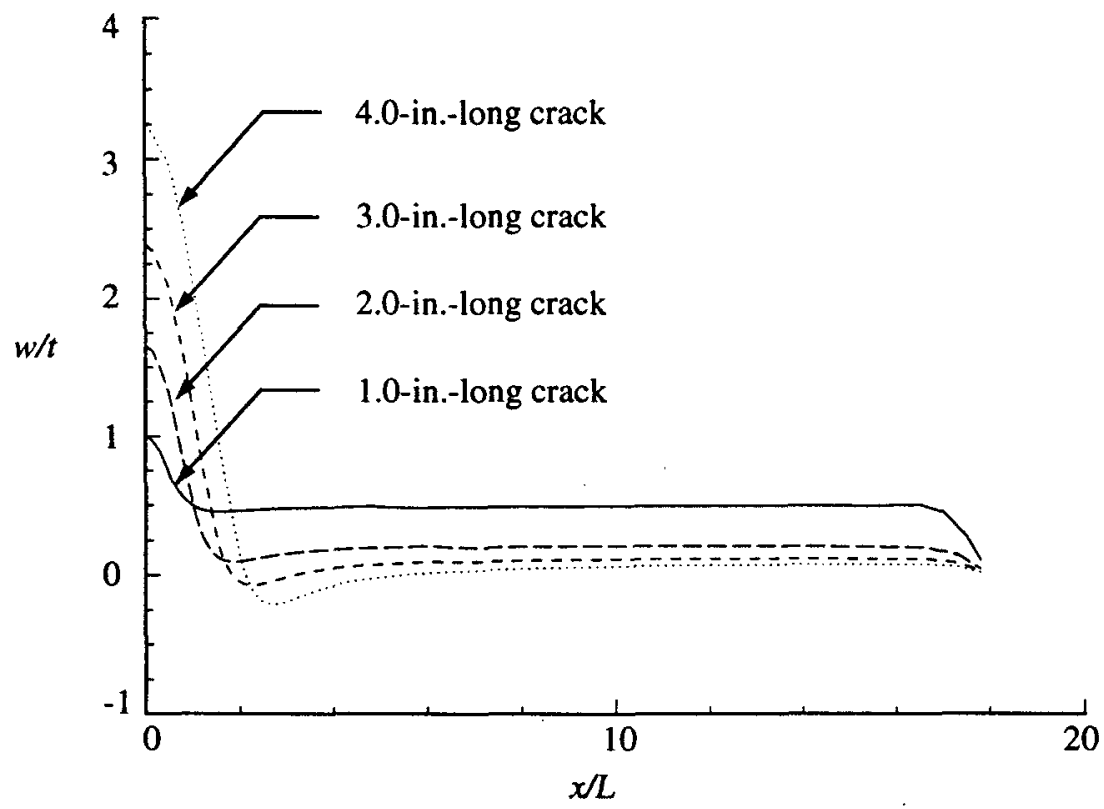

Figure 3. Radial displacement response along the shell length at the circumferential location $\theta=0^{\circ}$, just before the initiation of stable crack growth for 0.040 -inch-thick shells.

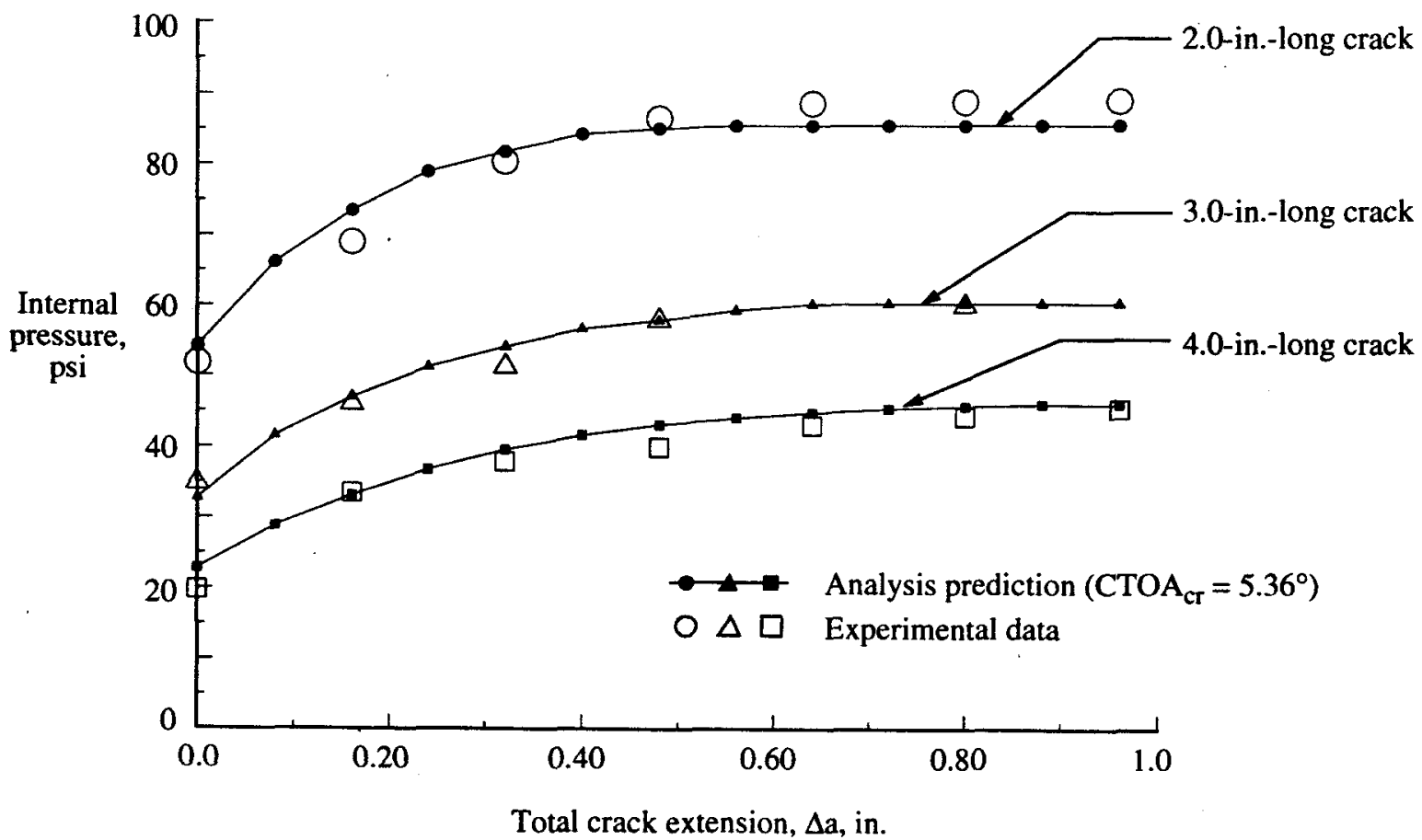

Figure 4. Comparison of analytical and experimental total crack extension results for 0.040 -inch-thick internally pressurized shells. 


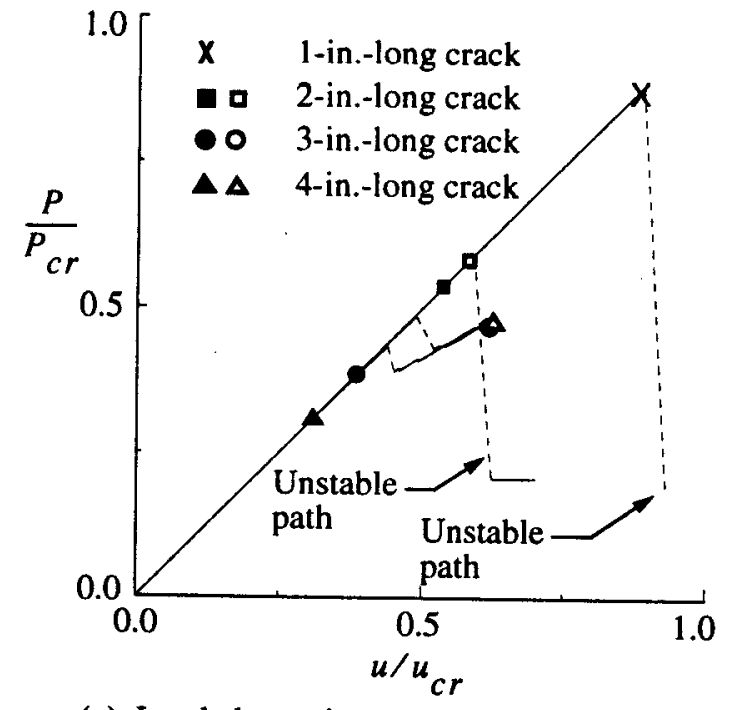

(a) Load-shortening response for 0.040-inch-thick shells

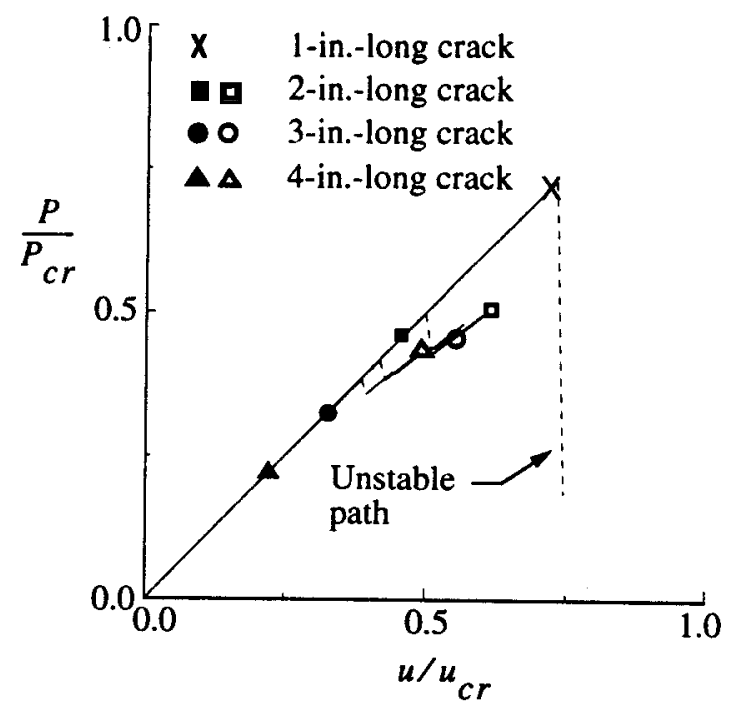

(b) Load-shortening response for 0.020 -inch-thick shells

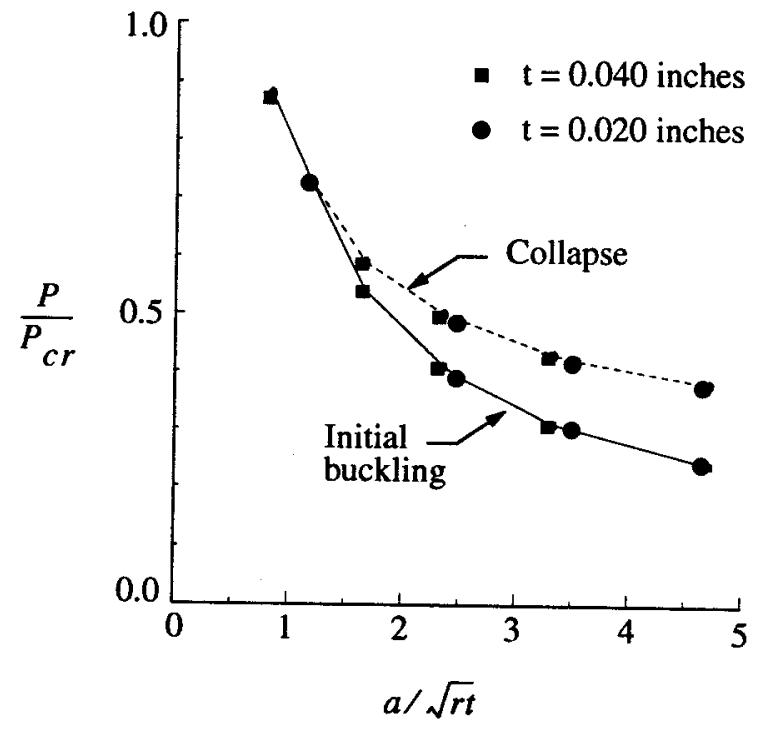

(c) Initial buckling and collapse loads Figure 5 . Effect of initial crack length on the response of cylindrical shells with longitudinal
cracks and subjected to axial compression. 


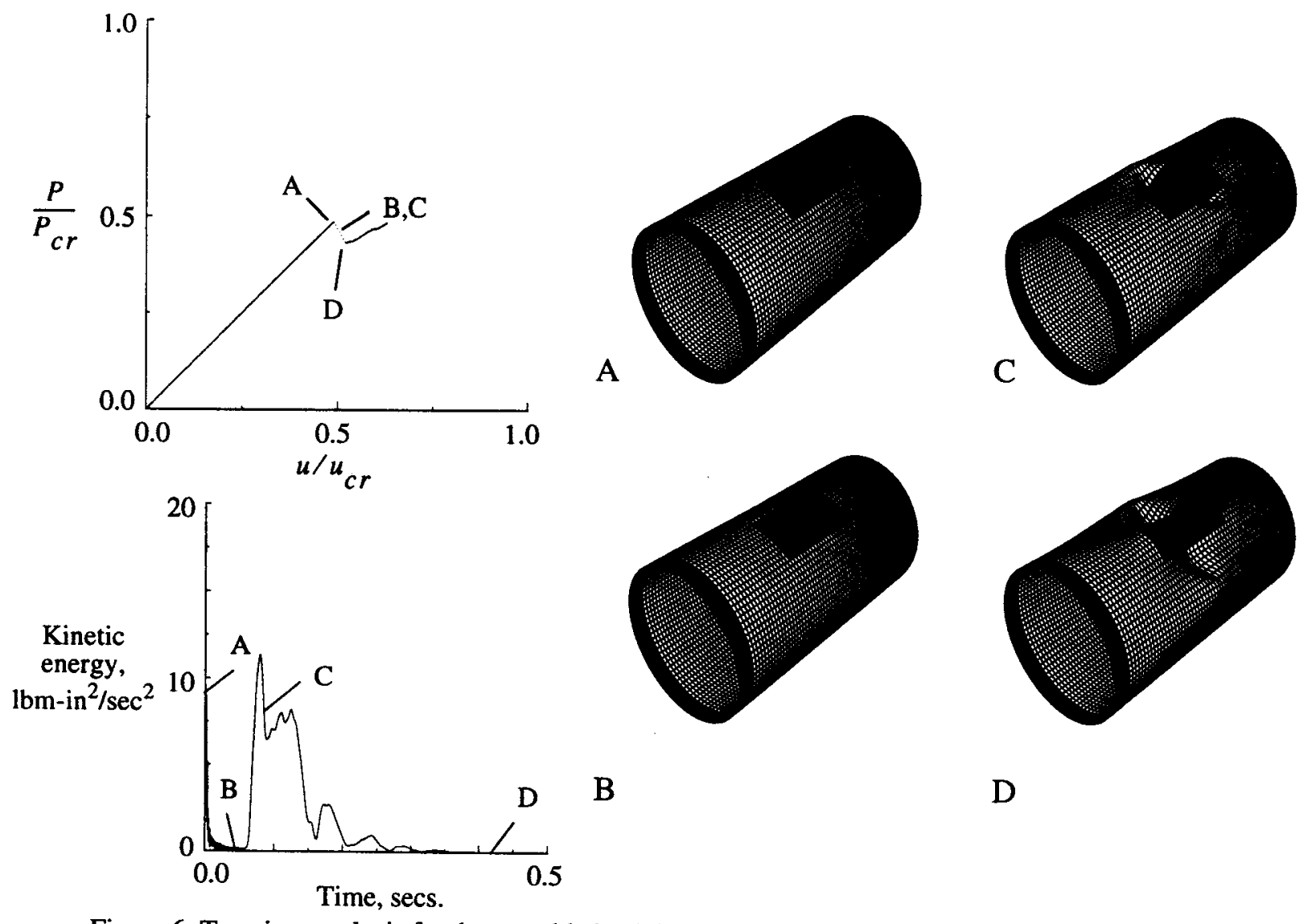

Figure 6. Transient analysis for the unstable buckling response of a 0.040 -inch-thick shell with a 3.0 -inchlong crack and subjected to axial compression.

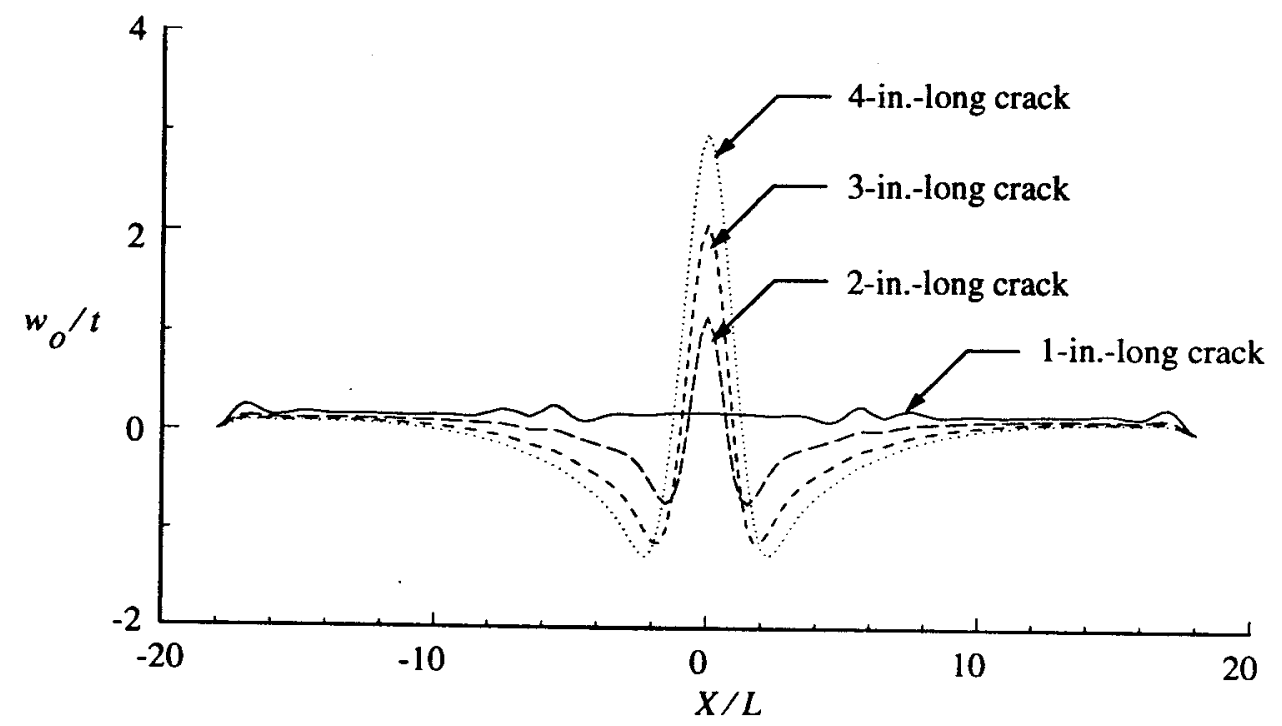

Figure 7. Prebuckling radial displacement response of 0.040 -inch-thick shells along $\theta=0^{\circ}$. 


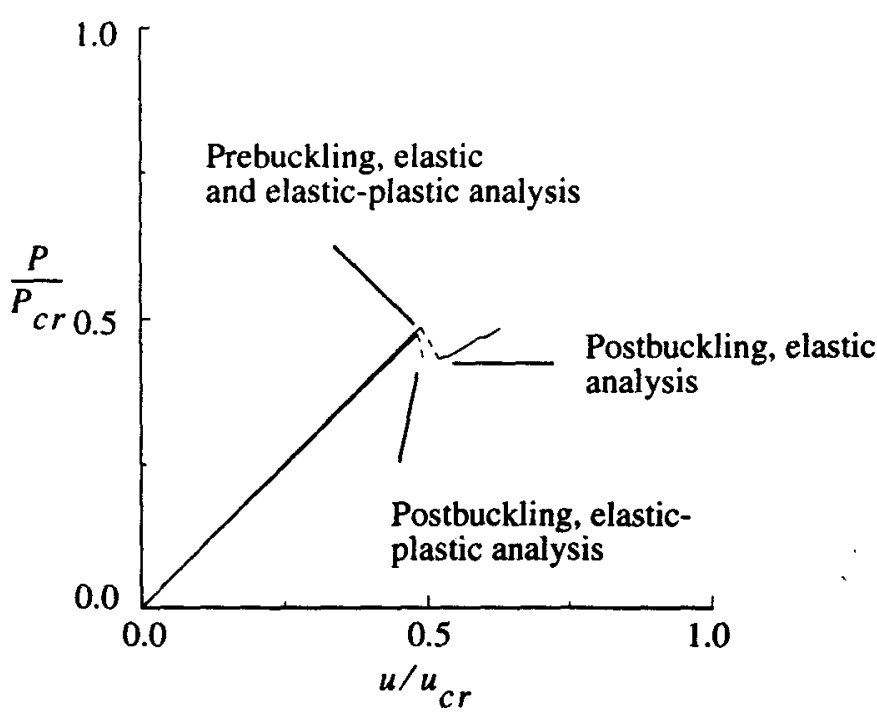

(a) Predicted load-shortening relation for linear-elastic response and material-nonlinear response

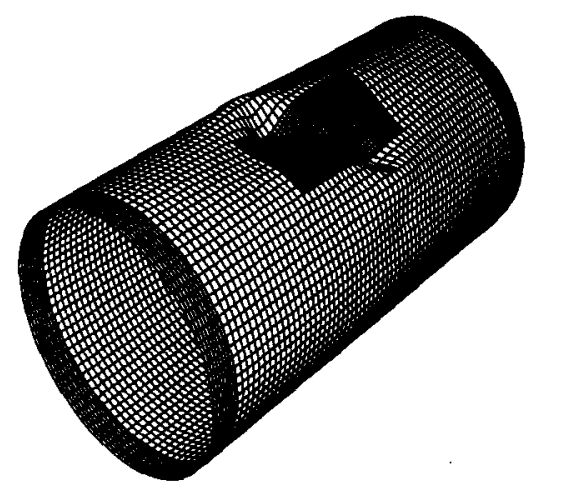

(c) Predicted initial postbuckling deformation pattern for material-nonlinear response

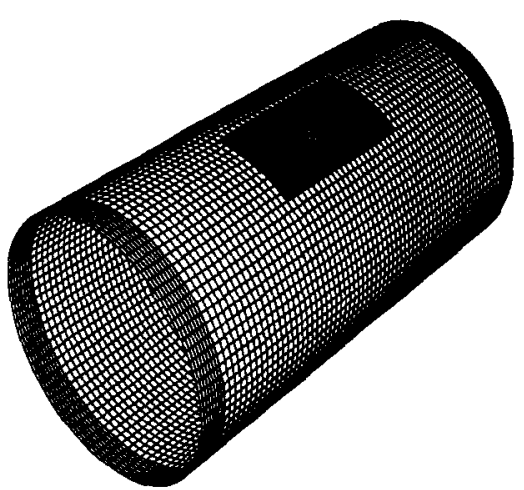

(b) Predicted prebuckling deformation pattern for linear-elastic response and material-nonlinear response

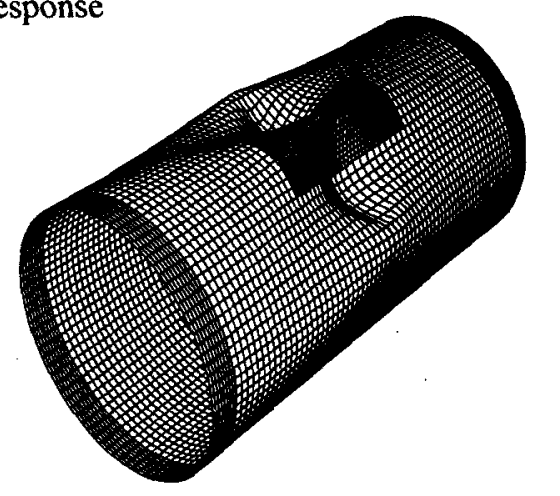

(d) Predicted initial postbuckling deformation pattern for linear-elastic response

Figure 9. Comparison of linear-elastic and material-nonlinear responses of a 0.040 -inch-thick shell with a 3.0-inch-long crack and subjected to axial compression. 


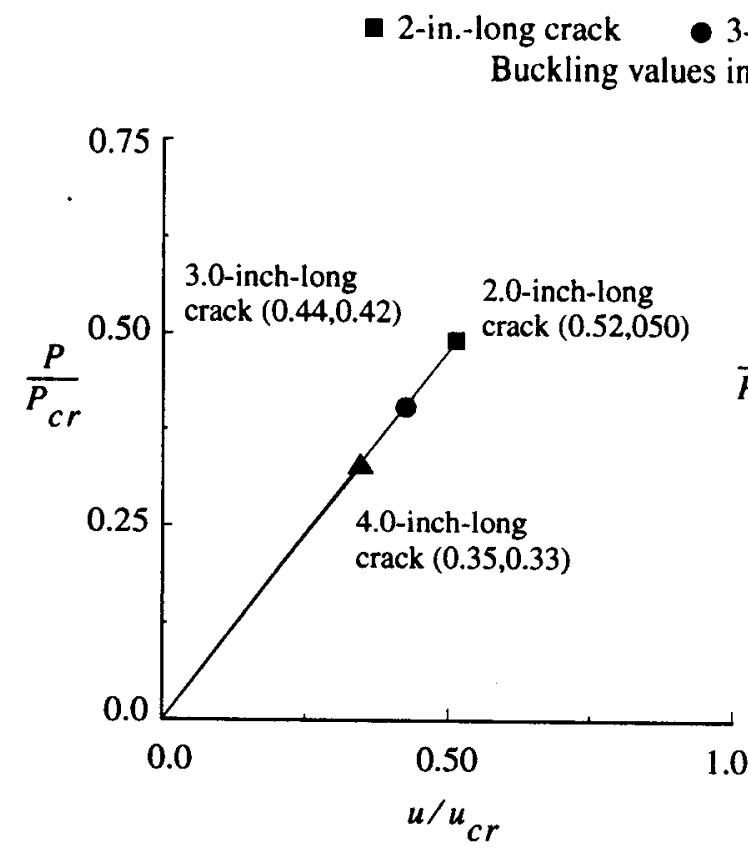

(a) Predicted prebuckling load-shortening relations 3-in.-long crack $\Delta$ 4-in.-long crack

in parenthesis: $\left(w / u_{c r}, P / P_{c r}\right)$

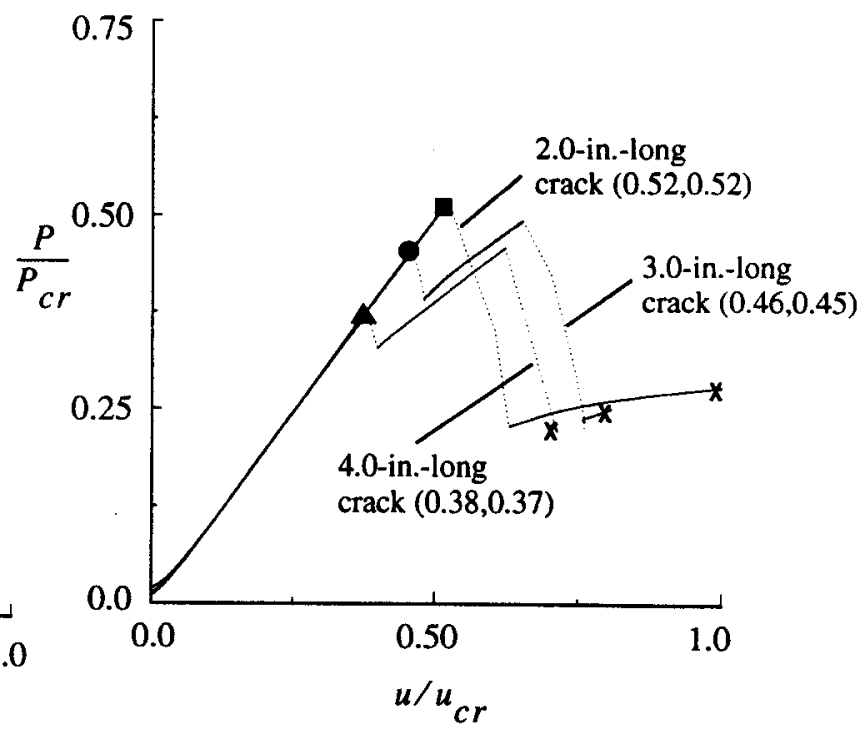

(b) Measured load-shortening relations

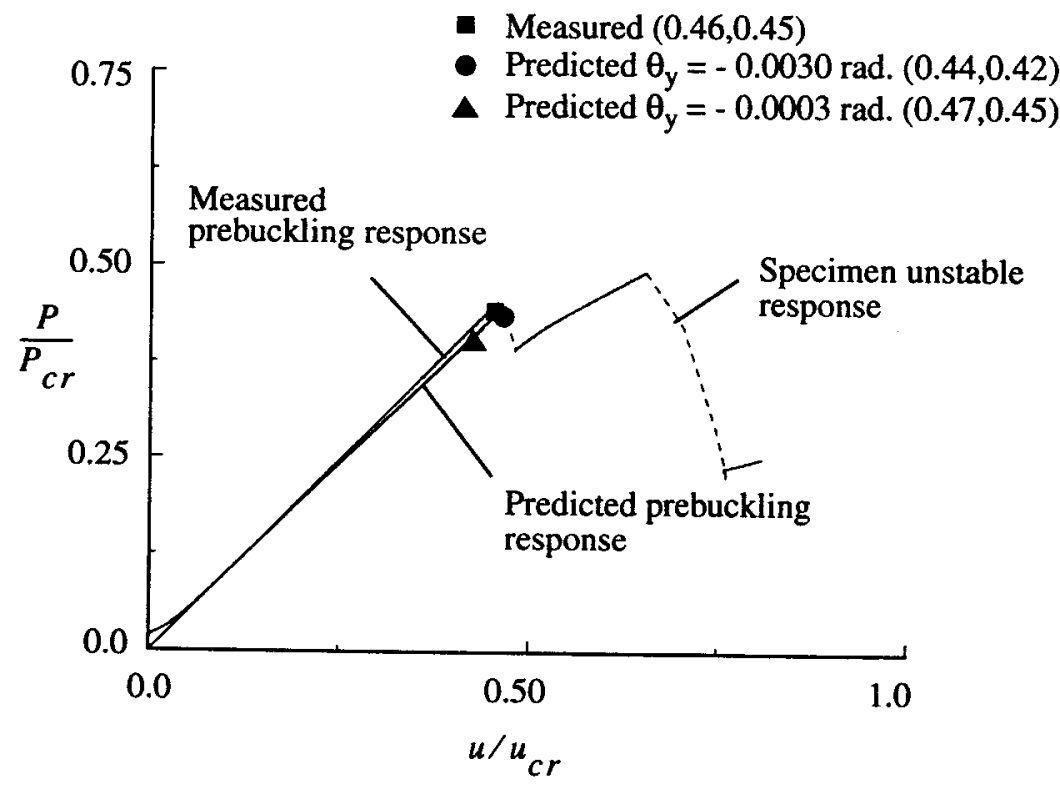

(c) Effect of end rotations on the load-shortening results for a shell with a 3.0 -inch-long crack

Figure 10. Summary of predicted and experimental load-shortening relations for 0.040 -inch thick shells subjected to axial compression. 